\title{
Localized and delocalized plasmons in metallic nanovoids
}

\author{
T. A. Kelf, Y. Sugawara, R. M. Cole, and J. J. Baumberg* \\ School of Physics and Astronomy, University of Southampton, Highfield, Southampton, SO17 1BJ, United Kingdom
}

M. E. Abdelsalam, S. Cintra, S. Mahajan, A. E. Russell, and P. N. Bartlett

School of Chemistry, University of Southampton, Highfield, Southampton, SO17 1BJ, United Kingdom

(Received 26 June 2006; revised manuscript received 4 October 2006; published 13 December 2006)

\begin{abstract}
Nanostructured metal films comprised of periodically arranged spherical voids are grown by electrochemical deposition through a self-assembled template. Detailed measurements of the angle- and orientation-dependent reflectivity for different sample geometries reveal the spectral dispersion of several different types of surface plasmon modes. The dependence of the energies of both delocalized Bragg and localized Mie plasmons on the void goemetry is presented, along with theoretical models to explain some of these experimental findings. Strong interactions between the different plasmon modes as well as other mixing processes are identified. Understanding such plasmonic crystals allows for the engineering of devices tailored for a wide range of sensing application.
\end{abstract}

DOI: $10.1103 /$ PhysRevB.74.245415

PACS number(s): 42.70.Qs, 73.20.Mf, 72.15.Rn, 81.07.-b

\section{INTRODUCTION}

The field of plasmonics is rapidly becoming of interest to a broad range of disciplines. ${ }^{1}$ Stemming from the desire to construct plasmon devices and the ability to make and characterize metallic structures on the nanoscale, plasmonics finds applications in fields as diverse as optical switching, ${ }^{2}$ near-field photonics, ${ }^{3}$ and surface-enhanced Raman spectroscopy. ${ }^{4}$ While still in its infancy, the field of plasmonics has already shown many interesting results including the study of full plasmonic band gaps ${ }^{1}$ as well as plasmonic waveguiding. ${ }^{5}$ Also of some contention has been the enhanced transmission of light through subwavelength apertures, ${ }^{6,7}$ which generally focus on a quasi-twodimensional geometry. Here we report an in-depth investigation of both surface and localized plasmons found on a highly controlled series of three-dimensional (3D) nanostructured metallic surfaces. Understanding the range of plasmon modes and their energies gives clear insights into how to optimally pattern such surfaces for particular plasmon modes.

Surface plasmons are electromagnetic waves coupled to charge fluctuations, and are pinned to the interface between a metal and a dielectric. On a flat surface a surface plasmon has a greater momentum than an optical field traversing above the surface, and so no optical coupling is allowed. However, when the surface is patterned, the optical field can scatter to higher-momentum states and excite a surface plasmon polariton-an optically active mixture of surface plasmon and optical fields. While most plasmonic surfaces to date have been based on a two-dimensional planar architecture, here we develop coupling in 3D in terms of plasmon "atomlike" states connected by a plasmon "bus." The threedimensional nanostructured surfaces containing voids are cast around arrays of spherical particles. By varying the film thickness, architectures ranging from arrays of shallow dishes to encapsulated voids are produced.

These structures are ideal for studying both localized and delocalized surface plasmons [Fig. 1(a)]. Surface plasmon polaritons (SPPs) exist on the sample top surface and have energies that depend strongly on both incident angle and sample orientation. The experimental dispersion of these delocalized plasmon modes is shown in Fig. 1(b), plotting photon energy against incident angle (and hence in-plane wave vector $k_{\|}=2 \pi \sin \theta / \lambda_{0}$ ) for the absorption of a beam of light reflected off the surface. The color scale represents the amount of absorption, and hence coupling into plasmon modes. In this paper these surface plasmon polaritons will be termed "Bragg plasmons" due to their coupling via Bragg scattering and to distinguish them from other plasmon oscillations. ${ }^{8}$ If a surface plasmon polariton wave is reflected

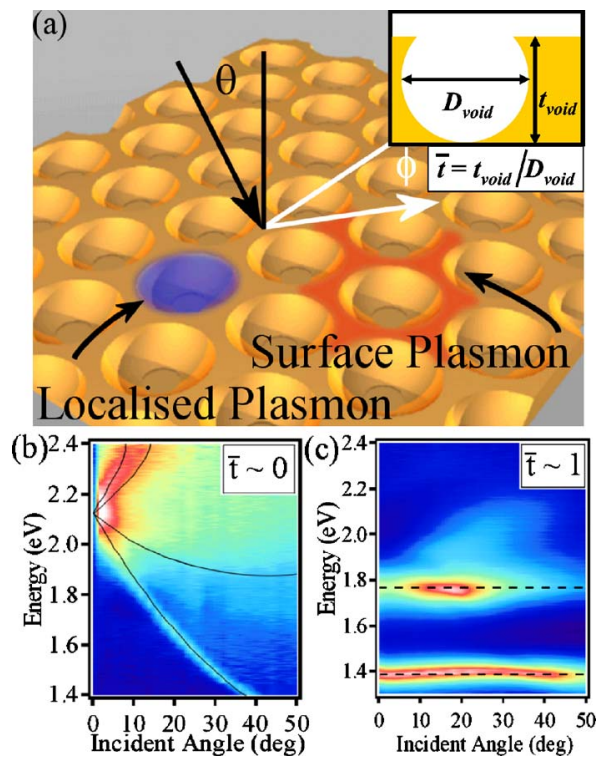

FIG. 1. (Color online) (a) Schematic of surface- and localizedplasmons on nanostructured gold surfaces. Inset shows definition of normalized thickness $\bar{t}$. (b) Typical dispersion of a Bragg plasmon; black lines indicate theoretical dispersion modeled using the Bragg scattering equation. (c) Mie plasmon dispersion; dashed black lines indicate guide to eye. Color (gray) scale in both images is blue (dark) for $0 \%$ plasmon absorption to white for $80 \%$ of incident light coupled into the plasmon mode. 
between barriers it will interfere with itself and form a standing wave, localizing the surface plasmon polariton. Localization also occurs around features with sharp asperities. For the structures studied in this paper this localization occurs within truncated spherical cavities, the electromagnetic solutions to which will be shown to relate to those of Mie scattering. For this reason these localized plasmons will be termed "Mie plasmons." Mie plasmons have energies that are highly dependent on the nanostructured geometry, although they are independent of the incident angle of the excitation light and sample orientation because they are localized [Fig. 1(c)]. Thus angle-resolved spectroscopy is the key to distinguishing the plasmons on such surfaces.

It is convenient to define the normalized sample film thickness to describe the different sample geometries. This is defined as $\bar{t}=t_{\text {void }} / D_{\text {void }}$, where $D_{\text {void }}$ is the diameter of the void and $t_{\text {void }}$ is the film thickness, so that the fully encapsulated void has $\bar{t}=1$.

The layout of this paper is as follows. After a brief description of the sample fabrication and optical arrangement (Sec. II), we discuss Bragg plasmons on thin samples (Sec. III). Following this, Mie plasmons are investigated (Sec. IV), and two intuitive models are proposed to understand the dependence of the Mie plasmon energy on film thickness. The final section (Sec. V) explores the interactions and mixing between the Bragg and Mie plasmons.

\section{NANOSTRUCTURED SAMPLES AND EXPERIMENTAL SETUP}

The nanostructured surfaces studied in this paper are produced via a template self-assembly and electrochemical deposition technique. ${ }^{9,10}$ In this process, a solution of polymer spheres fills a thin fluid cell, which is made of a goldcoated glass slide, a clean glass slide, and sidewalls made from a thin $300 \mu \mathrm{m}$ spacer layer of Parafilm. The gold slide is coated in cysteamine to reduce the contact angle of aqueous liquid placed on the surface. This forms a sweeping meniscus tail as the fluid dries, which pulls spheres to the evaporation line where they form a close-packed monolayer domain. ${ }^{10}$ Using this technique, monolayer micro-sphere array areas greater than $1 \mathrm{~cm}^{2}$ can be produced. After template formation the sample is placed in an electrochemical plating bath. By monitoring the current passing through the bath, the thickness of metal deposited on the surface can be accurately controlled. The plated metal fills the interstitial regions between the microspheres, and thus creates a cast of the initial template. After plating, the sample is washed in tetrahydrofluoran for several hours to completely remove the initial template. To date, metal structures made from gold, silver, copper, platinum, palladium, and nickel have been produced, although in this paper only gold samples will be discussed (due to their strong plasmon properties and their chemical inactivity, which removes any issues about stability). Results for silver films are essentially identical, while most of the plasmon response is absent in platinum and nickel films. Stopping the plating process at a given thickness provides control over the final geometry of the sample. Furthermore, by systematically retracting the sample from the plating so-

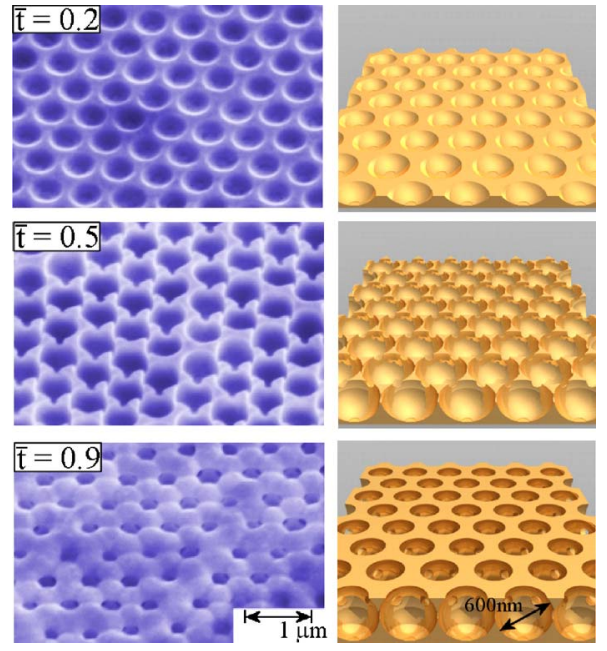

FIG. 2. (Color online) Scanning electron microscope images of a sample with a void diameter of $600 \mathrm{~nm}$ at three normalized thicknesses $\bar{t}=0.2,0.5$, and 0.9 . Sample is tilted by $45^{\circ}$ for clarity of viewing. Schematic structures of the surface at each thickness are also shown.

lution during growth, various geometries can be constructed on a single sample. Three such positions, on a sample made from 600-nm-diameter voids, are shown in Fig. 2.

When the plated gold layer is very thin the surface takes the form of an array of shallow dishes, ordered in a closepacked lattice. As the thickness of the gold film increases to $\bar{t}=0.5$, triangular islands form between the truncated spherical cavities. With increasing thickness these islands connect and the film grows over the spheres to form encapsulated spherical cavities. Due to the different growth rate between the spheres in the template, the top surface is not flat for $\bar{t}$ $>0.5$ but forms a hexagonal array of mounds surrounding the entrance to each cavity. The precise control over surface geometry allows the study of many different plasmon architectures along with the different interactions between different plasmons.

The dispersion of the plasmons is investigated by recording the reflectivity spectrum for different incident angles $\theta$ and sample azimuthal orientations $\phi$ and for various sample thicknesses $\bar{t}$ and hence geometries. A supercontinuum white-light laser is used to simultaneously study absorption features throughout the visible and infrared spectral regions. Samples are mounted on a custom-built computer-controlled goniometer. This can be programmed to move in both the $\theta$ and $\phi$ directions as well as move in the $x-y$ plane of the sample. An optical fiber is attached to the end of the goniometer arm, on which the reflected light is focused, and this is also positioned under computer control. The light is polarized before illuminating the sample and a polarizer is placed before the fiber to select the polarization of the reflected light. Spectra are collected using both visible and infrared spectrometers (Ocean Optics USB2000 and NIR512), also controlled via the same software used to operate the goniometer. This setup can therefore perform reflectivity measurements over a wide range of parameters. The data collected are then combined into a four-dimensional matrix 
$R(\theta, \phi, \bar{t}, \lambda)$, where they can be viewed and analyzed. One minor limitation with the current setup is the requirement for the light reflected off the sample to pass under the mirror directing the incident light onto the sample. While this arrangement provides reflected incident angles from $90^{\circ}$ to $-30^{\circ}$ to be recorded, the light is not quite completely in plane. We define the incident angle in the $x$ plane, $\theta$, and note that our data are taken with an angle of $\theta_{y}=2^{\circ}$ in the orthogonal direction. This angle leads to a small splitting of the observed SPP modes, a factor that is also accounted for theoretically when considering the data.

\section{DELOCALIZED PLASMONS}

\section{A. Thin samples}

For sample regions where $\bar{t}<0.3$ the nanostructured surfaces take the form of a thin two-dimensional grating. A six-fold diffraction pattern from these structures is observed, the energetics of which fit well with that of a weak-scattering approximation. This model assumes the surface to be flat with point scattering of a certain periodicity in a hexagonal lattice. In this 2D array two lattice vectors $\mathbf{a}$ and $\mathbf{b}$ parametrize the scattering sites. These vectors are orientated at $60^{\circ}$ to one another and have a magnitude equal to the center-tocenter spacing of the dishes. Under ideal sample growing conditions this periodicity would be equal to the void diameter; however, due to the templating process the actual domain pitch is found to be $50 \mathrm{~nm}$ greater than the diameter of the voids. The close-packed nature of the surface leads to diffraction from the planes of scatters, which are spaced by a distance $\boldsymbol{\Lambda}=\sqrt{\frac{3}{4}}(m \mathbf{a}+n \mathbf{b})$, where $m$ and $n$ are integers.

Bragg plasmons can be modeled using the weakscattering approximation with the energy of a surface plasmon mode, $E(\theta, \phi)$, given by

$$
E(\theta, \phi)=\hbar c \sqrt{\epsilon_{m}^{-1}(E)+\epsilon_{d}^{-1}} f(\phi)\left|\mathbf{k}_{0} \sin \theta+\mathbf{q}_{n m}\right|
$$

where $\mathbf{k}_{0}$ is the wave vector of the incident light, $\mathbf{q}_{m n}$ $=2 \pi / \Lambda_{m n}, \epsilon_{d}$ is the dielectric constant of the region above the surface, and $\epsilon_{m}(E)$ is the energy-dependent complex dielectric constant of gold. ${ }^{11}$ Finally $f(\phi)$ is a function that accounts for the dependence in sample orientation of the ingoing beam on the sixfold symmetric surface. ${ }^{16}$ The solutions to Eq. (1) represent all allowed plasmon modes which can be coupled from the incident beam $\mathbf{k}_{0}$, giving at any wavelength a maximum of six solutions for the lowest bands. This compares to diffraction where light of any wavelength shorter than $|\boldsymbol{\Lambda}|$ can be diffracted from the surface. Thus there will be a reduction in reflection as diffraction turns on for $\lambda<|\boldsymbol{\Lambda}|$. It is found that the redistribution of light into the diffracted orders is around 100 times weaker than the absorption from the surface plasmon modes in our structures, and hence the diffracted features are not visible in the data. This is due to the low aspect ratio of the surface morphology reducing the overall diffraction efficiency, and is a general result on all samples studied. Hence the effect of diffraction can be neglected throughout the remainder of this paper.

The wave vectors of Bragg plasmons (red) excited by the coupling between the smallest reciprocal lattice vectors $\mathbf{q}_{m n}$

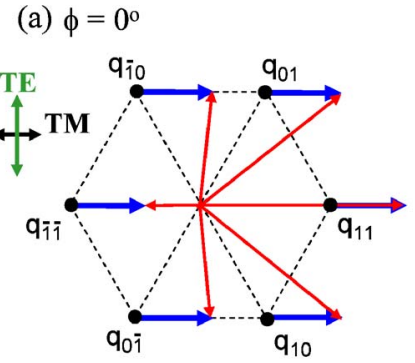

(b) $\phi=30^{\circ}$

(c) $\phi=0^{\circ}$
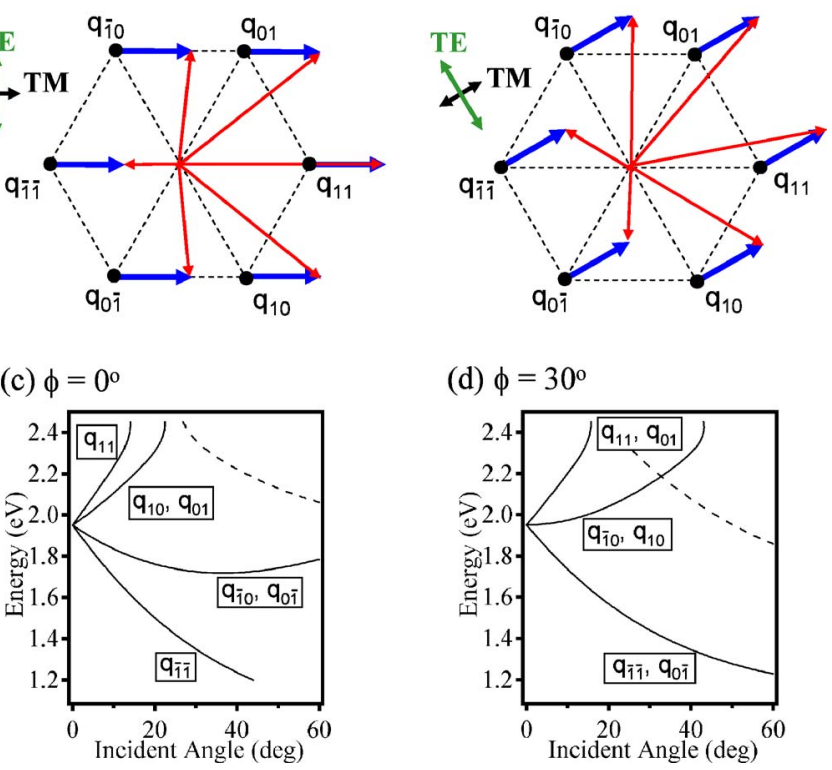

(d) $\phi=30^{\circ}$

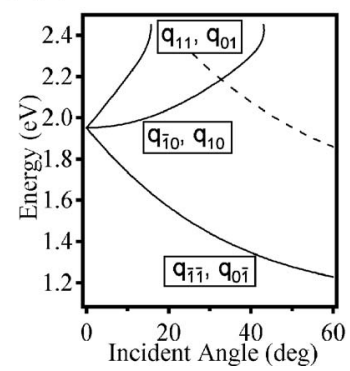

FIG. 3. (Color online) Bragg plasmons excited by the coupling to smallest reciprocal lattice vectors $\mathbf{q}_{m n}$ for in-plane components of incident light with $\phi=0^{\circ}$ and $30^{\circ}$. (a), (b) Wave vectors of Bragg plasmons [red (gray) arrows] coupled by incident in-plane $\mathbf{k}_{\|}$[blue (dark) arrows]. (c), (d) Theoretical Bragg plasmon energies as a function of incident angle calculated from Eq. (1) for void separation of $700 \mathrm{~nm}$. The broken lines show plasmon bands excited by the next-nearest wave vectors.

for in-plane components of incident light with $\phi=0^{\circ}$ and $30^{\circ}$ are shown in Figs. 3(a) and 3(b). This leads to theoretical Bragg plasmon dispersions as a function of incident angle calculated by Eq. (1) [Figs. 3(c) and 3(d)]. Each band labeled with $\mathbf{q}_{m n}$ corresponds to a plasmon band excited by diffraction from a reciprocal vector $\mathbf{q}_{m n}$ in Figs. 3(a) and 3(b). Plasmons propagating in different directions have different energies at the same incident angle in these dispersion relations even though the SPP dispersion is isotropic, due to the geometry of the optical interaction. Generally Bragg plasmons are excited in six in-plane directions at six different energies if unpolarized light is incident on the sample. However, degeneracies at symmetry orientations of $\phi=0^{\circ}$ and $30^{\circ}$ reduce the number of modes to 4 and 3 , respectively [as apparent in Figs. 3(c) and 3(d)]. In addition, observation of Bragg plasmon modes depends on the polarization of the incident light because SPPs are allowed only in TM polarization. This results in different coupling efficiencies for different incident light polarization and plasmon direction.

This model can be superimposed on experimental data of angle-resolved reflectivity on a sample with void diameters of $700 \mathrm{~nm}$, and thickness $\bar{t}=0.29$ (Fig. 4). Both TM (left) and TE (right) polarized data are superimposed for comparison of the coupling to the plasmon dispersion. The broken lines show the predictions from Eq. (1) using the SPP dispersion. A clear correlation between experiment and theory for the lowest modes can be observed using the SPP dispersion, while the upper bands are more obscured by increased attenuation from the 2D SPPs below $\lambda=500 \mathrm{~nm}$ for Au.

The propagating SPP Bragg plasmons are more clearly resolved by taking cuts through the TM-polarized data at 


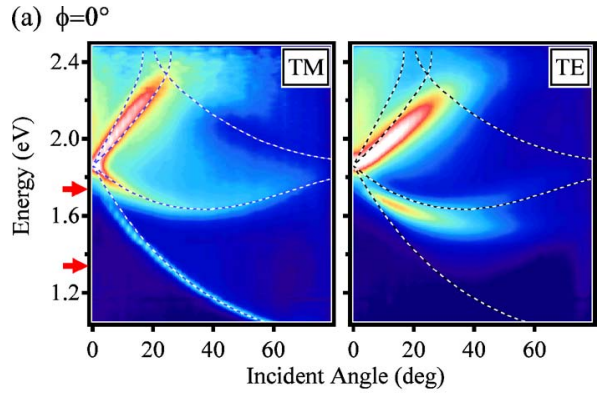

(b) $\phi=30^{\circ}$

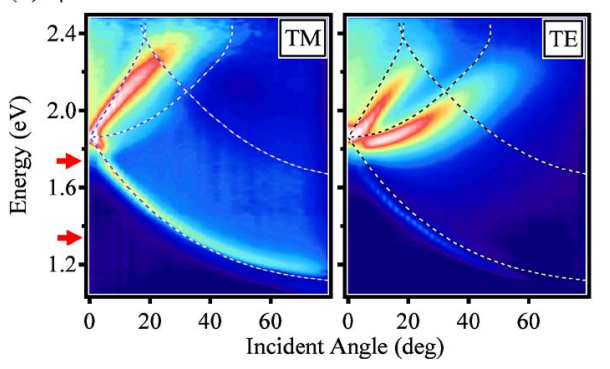

FIG. 4. (Color online) Reflectivity of a surface with void diameter $700 \mathrm{~nm}$ at a thickness of $\bar{t}=0.29$ for $\phi=$ (a) $0^{\circ}$ and (b) $30^{\circ}$. Plots show strength of reflected TM- (left) and TE- (right) polarized light at different photon energies and incident angles. Lines show theory for Bragg plasmons, as well as cuts taken for Fig. 5 [(red) arrows]. Data are on a logarithmic scale.

specific energies, and matching the energy surfaces in wave vector space $\left(k_{x}, k_{y}\right)$ to those expected from the folded bands of Eq. (1). Two such cuts are shown in Fig. 5, together with theoretical calculations using the empty lattice approximation, showing the excellent agreement with the first folded bands and the six-fold symmetry of the SPP dispersion in the close-packed lattice. Near the $\Gamma$ point, the simple dispersions are perturbed by the coupling between the different modes (plasmonic band gaps discussed below).

By using both types of image cuts $R(\theta, \phi)$ and $R(\theta, E)$ from the four-dimensional data set $R(\theta, \phi, E, \bar{t})$, it is thus possible to identify and characterize the Bragg plasmon modes. This analysis shows that a weak-scattering approximation is a useful model in the plasmon dispersion when the sample is thin $(\bar{t}<0.3)$.

\section{B. Pitch dependence}

By selecting spheres of different sizes to form the initial template it is easy to change the pitch of the final nanostructured surface. This can be used to tune the surface plasmon dispersion across the entire visible and infrared spectrum. The dispersions of four different samples with void diameters between 500 and $900 \mathrm{~nm}$ at $\bar{t}=0.1$ are shown in Fig. 6 . The predicted dispersion is overlaid on the data, and again shows a strong agreement between theory and experiment. For each sample the precise pitch is ascertained through scanning electron microscope (SEM) analysis, and this corrected pitch is used for the theory lines in Fig. 6. Similar observations on arrays of square-symmetry pyramidal pits with pitches up to $6 \mu \mathrm{m}$ also show corresponding features as well as higher-order bands. ${ }^{12}$
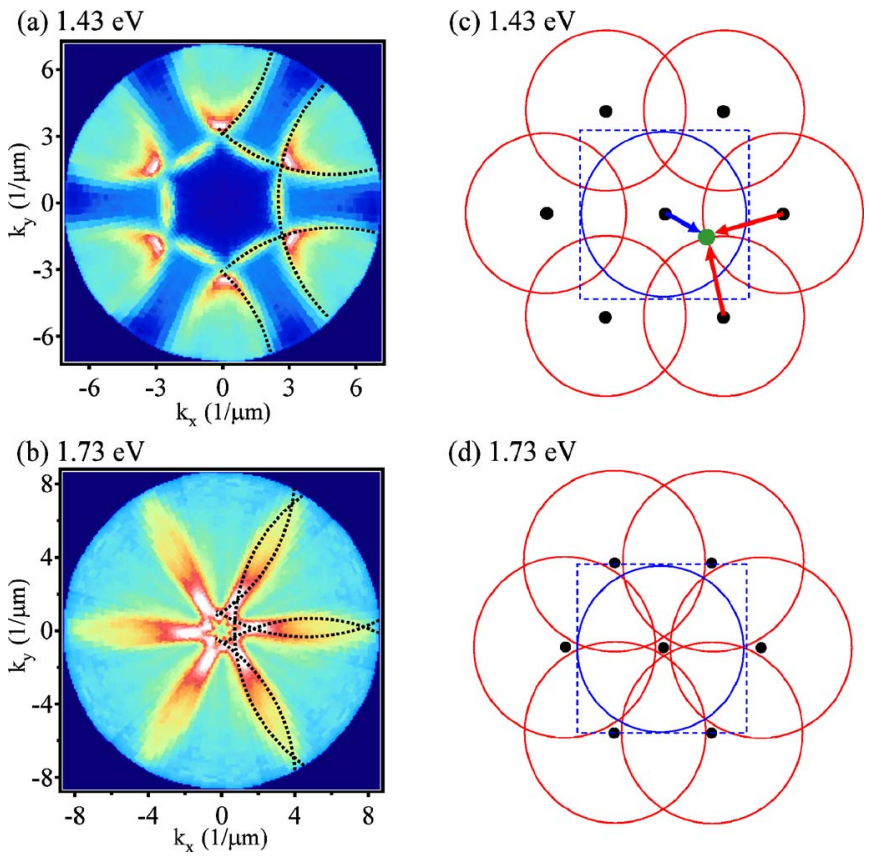

(d) $1.73 \mathrm{eV}$

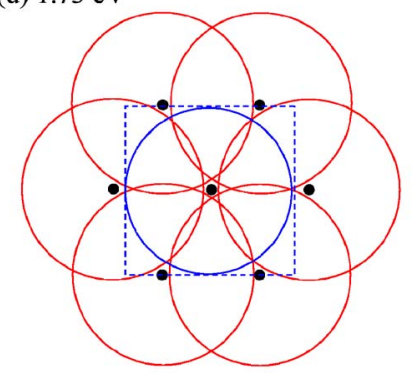

FIG. 5. (Color online) (a), (b) Reflectivity of the sample shown in Fig. 4 at $\hbar \omega=1.43$ and $1.73 \mathrm{eV}$ in $\left(k_{x}, k_{y}\right)$ in-plane wave vector space. The angles in the $\theta_{x, y}$ direction are recorded from $0^{\circ}$ to $80^{\circ}$, while the lines show Bragg plasmon theory. (c), (d) Folded bands in $\left(k_{x}, k_{y}\right)$ for the empty lattice approximation at these energies, for data in (a), (b).

\section{LOCALIZED PLASMONS}

\section{A. Plasmons in spherical voids}

When the sample thickness approaches $\bar{t}=1$, the surface structure takes the form of spherical cavities. The electromagnetic solutions to an isolated spherical dielectric cavity within an infinite expanse of metal correspond to Mie scattered modes from a sphere. ${ }^{13}$ These are calculated from Maxwell's equations expanded in spherical coordinates, by
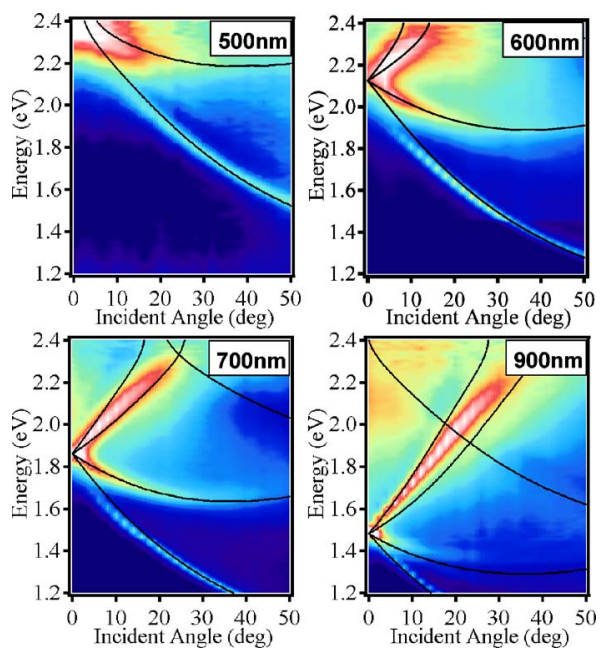

FIG. 6. (Color online) Dispersion plots at $\bar{t}=0.1$ and $\phi=0^{\circ}$ for samples with void diameters 500, 600, 700, and $900 \mathrm{~nm}$. Lines show theory for Bragg plasmons. 

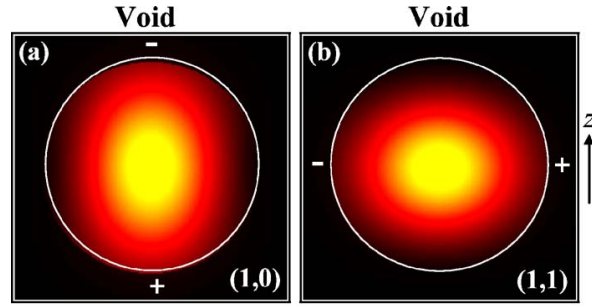

Void

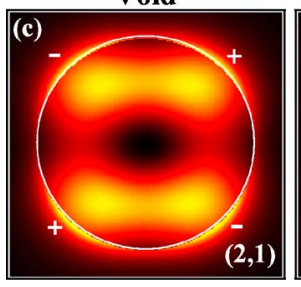

Particle

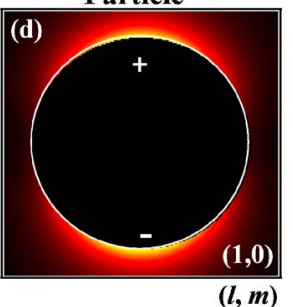

FIG. 7. (Color online) Computed electric field distributions for Mie plasmon modes in perfect spherical structures of (a)-(c) air void within an infinite gold medium and (d) a gold particle within an infinite air medium for specific $(l, m)$, symmetric about the $z$ axis.

matching boundary conditions at the sphere surfaces, and involve spherical Bessel, $J_{l}$, and Hankel, $H_{l}$, functions: ${ }^{14}$

$$
\epsilon_{d} H_{l}\left(k_{m} a\right)\left[k_{d} a J_{l}\left(k_{d} a\right)\right]^{\prime}=\epsilon_{m}(E) J_{l}\left(k_{d} a\right)\left[k_{m} a H_{l}\left(k_{m} a\right)\right]^{\prime} .
$$

Here, $a=D / 2$ corresponds to the void radius, $l$ is the (integer) index denoting the angular momentum, $k_{m}=\sqrt{\epsilon_{m}} k_{0}$ and $k_{d}=\sqrt{\epsilon_{d}} k_{0}$ are wave vectors in metal and void, and the prime indicates differentiation with respect to the argument $k a$. The lowest two mode solutions to this equation with $l=1,2$ are shown in Figs. 7(a)-7(c), for different azimuthal quantum numbers $m$.

These modes, which have not been carefully investigated previously, take the form of dipole $(l=1)$ and quadrupole $(l$ $=2$ ) charge oscillations within the metal. One interesting feature of the field in voids is that the $l=1$ mode has a singlelobed intensity distribution with $s$-like symmetry in spite of the $p$-like dipole charge oscillation. This is quite different from the $l=1$ localized plasmon mode for metallic balls, which has a traditional $p$-like two-lobed intensity distribution [Fig. 7(d)]. In general, the field distribution for localized plasmons in the void has a symmetry similar to the spherical harmonic function $Y_{(l-1, m)}$. While the $l=0 s$ state does not exist for plasmons on either solid sphere or void, it is approximately matched by the $l=1 p_{x, y, z}$ plasmons in the spherical void only. Plasmon modes are increasingly confined to the metal surface as $l$ increases, approaching surface plasmons propagating around the curved metal which resemble whispering gallery modes. Thus spherical voids combine the properties of plasmons on metal spheres with whispering gallery modes on high-refractive-index dielectric spheres, and their modes cannot be classified as either one or the other.

Using Eq. (2) allows the resonant energies of Mie localized plasmons to be computed in gold-encapsulated spherical

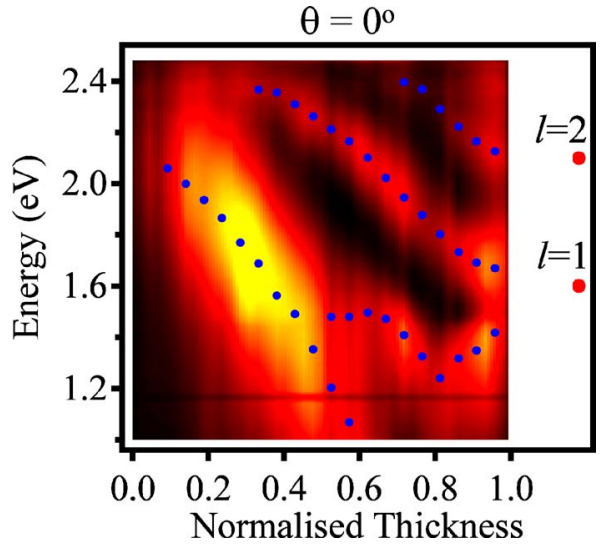

FIG. 8. (Color online) Reflectivity of a sample of randomly arranged voids with diameters equal to $600 \mathrm{~nm}$. Data are taken at normal incidence for voids of different thickness $\bar{t}$. (Red) points show calculated Mie plasmon energies in an encapsulated gold void; (purple) line is a guide for the eye.

voids. As we have previously shown, the energies of the void modes are blueshifted in comparison to the SPP modes on flat $\mathrm{Au}$, in contrast to redshifts for gold spheres. ${ }^{14}$ This allows voids to be strongly coupled to incident plane waves, even for large diameters (in the retarded field regime), in contrast to metal spheres. Together with their structural stability within a film (rather than free floating in solution), this leads to their effective use as plasmon substrates. However, to inject light into the voids, they must be brought to the surface of the metal, as we now discuss.

\section{B. Plasmons in truncated voids}

\section{Random voids}

In order to focus our discussion of the Mie modes in truncated spherical voids, data are first presented on a sample with randomly arranged $600 \mathrm{~nm}$ voids (also thickness graded) for which no Bragg modes are present (Fig. 8). Reflectivity at normal incidence as a function of thickness allows the Mie plasmons to be tracked in different goemetries. Note that because of the 7\% surface coverage of voids the absorption is considerably reduced. Also shown at $\bar{t}=1[(\mathrm{red})$ points] are the calculated $l=1,2$ Mie plasmons for the fully encapsulated perfect spherical void in gold.

Clearly evident is the increase in Mie plasmon energy as the void is progressively truncated. This is expected since the limit for $\bar{t} \rightarrow 0$ is the 2D SPP on flat Au at $\hbar \omega=2.4 \mathrm{eV}$. Truncating the void mixes the different multipolar plasmons, increasing their energies as they are increasingly drawn down to the metal surface. The modes at large thickness do not exactly match the calculated Mie modes, for a combination of reasons. In our structure, there are small holes into the void (Fig. 2) even around $\bar{t}=1$ due to the faster electrochemical growth between the voids (though this can be modified using surfactants). These holes lead to a drop in mode energy because of the reduced confinement, as recently shown theoretically by Teperik et al. ${ }^{15,16}$

The Mie mode solutions also imply that truncation will act to split the different symmetry $(l, m)$ plasmons. For in- 

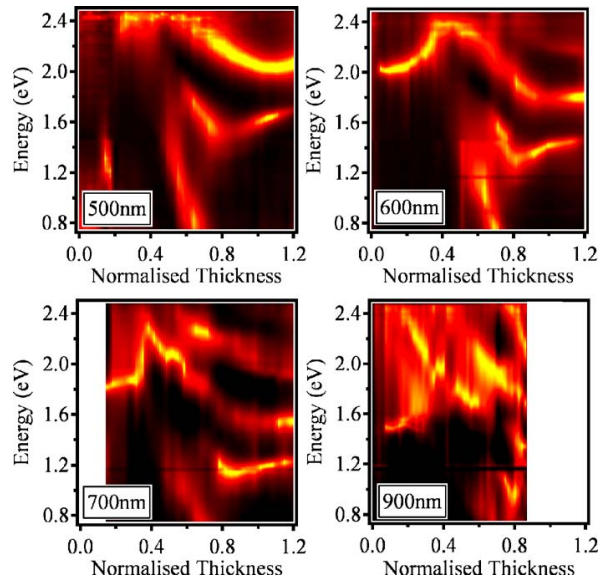

FIG. 9. (Color online) Mie plasmon absorption for samples with void diameters of 500,600, 700, and $900 \mathrm{~nm}$, showing plasmon mode energy vs $\bar{t}$ at normal incidence.

stance, the $l=1$ state will be split because the $p_{x, y}$ states [Fig. 7(b)] have strongest charge distributions around the equatorial diameter in the $x, y$ plane while the $p_{z}$ state [Fig. 7(a)] induces charge directly within the metal over the void which is truncated. Thus the $p_{z}$ state is expected to shift to higher energy at small truncations $1-\bar{t}$, while the $p_{x, y}$ modes drop in energy because of the increased radiative coupling. Similar considerations for the $l=2 d$ state, which is split into three bands, produce the complex behaviour observed in Fig. 7(c).

\section{Periodic voids}

On periodic arrays of voids, these Mie plasmons can also be identified, together with the Bragg plasmons. Absorption spectra $(\alpha=1-R$ when diffraction is negligible as here) at normal incidence for graded thickness samples of void diameter 500, 600, 700, and $900 \mathrm{~nm}$ are shown in Fig. 9. The same clear trend of the Mie plasmons is seen for $\bar{t}>0.5$. However for $\bar{t}<0.5$ the mixing with Bragg plasmon modes results in a more complex dispersion.

In order to identify the energy scaling with void diameter, it is possible to collapse this data onto a universal plasmon dispersion. A renormalization of the mode energy is used which combines the scaling with void diameter, $D$, of the experimental Mie modes, $E_{l}(D)$, at large thickness, with the independence of plasmon energies on the flat surface $(\bar{t}$ $\rightarrow 0$ ):

$$
E_{l}^{\prime}(D)=\left[E_{l}(D)-E_{s}\right]\left(\frac{600 \mathrm{~nm}}{D}\right)+E_{s}
$$

where $E_{s}=2.8 \mathrm{eV}$ is a scaled 2D SPP energy, and only the energy difference from $E_{s}$ is scaled by a function of the sphere diameter (referenced to $D=600 \mathrm{~nm}$ ). Above $\bar{t}=1$ the scaling is clamped due to the almost complete encapsulation of the voids.

Both the experimental Mie plasmon modes and the renormalised Mie mode energies are plotted in Fig. 10. This shows that the localized plasmon dispersions on truncated voids follow universal curves that can be identified with dif-
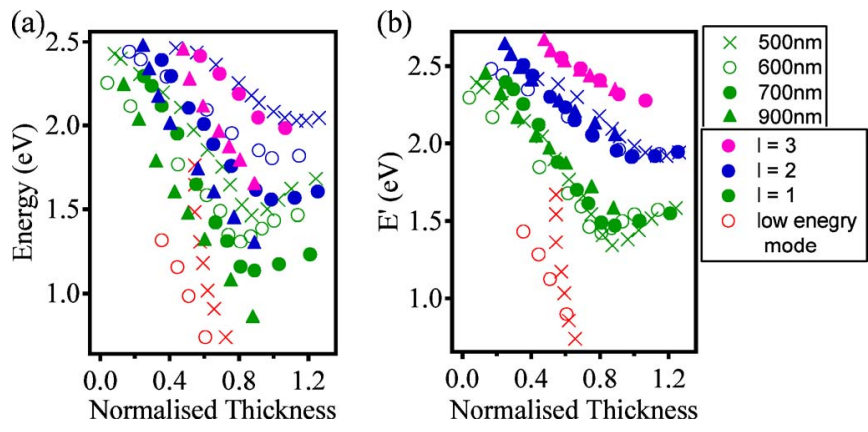

FIG. 10. (Color online) Extracted Mie plasmon absorption energies for graded samples with void diameters of 500,600, 700, and $900 \mathrm{~nm}$ as a function of void thickness $\bar{t}$, for (a) raw data and (b) renormalized energies. Marker type indicates void diameter; marker color indicates mode angular momentum.

ferent angular momenta $l$. The additional lowest-energy mode (red) arises from an unusual morphology near $\bar{t}=0.5$ and will be discussed in Sec. V. Thus, while it is not yet possible to theoretically calculate localized plasmon energies in truncated voids, experimentally these modes can be clearly mapped and their dependence on $(\bar{t}, D)$ understood in an effective way.

\section{Simple localized plasmon models}

As discussed, analytic solutions of Maxwell's equations for truncated voids are not (yet) possible. While Mie models give some guidance to the mode properties, it is possible to develop two additional models that give insight into the observed energetics of the Mie plasmon modes. The first model uses a ray optics approach to describe the optical field within the cavity. In this model resonance conditions arise from the interference of different ray paths through the cavity. The second model is based on a standing plasmon wave, assumed to be pinned to the surface of a two-dimensional void. Both models show similar trends to that observed in the data and are of interest in building an intuitive understanding.

\section{Ray optics model}

The universal scaling of the Mie plasmon modes within voids that are both larger and smaller than the wavelength of the absorbed light has been shown in Fig. 10. We are guided to a ray optics approach by our previous work treating the polarization dependence of shallow $2.5 \mu \mathrm{m}$ radius-ofcurvature cavities. ${ }^{17}$ Although the ray approximation should break down when the structures become smaller than the wavelength of light, surprisingly, no obvious change in the experimental optical response is observed as the structures pass through this size scale. In Fig. 10(b), wavelengths $\lambda$ $\sim D=500$ to $900 \mathrm{~nm}$ correspond to $E=2.48$ to $1.38 \mathrm{eV}$, respectively, and so corrections to this model must feed in slowly with decreasing $D / \lambda$.

We consider a three-dimensional cavity struck by bundles of rays onto the surface at an angle $\theta_{i}$ (Fig. 11). All possible reflections are considered and a set of ray paths is produced. Only rays leaving the surface in the reflected direction $\theta_{r}$ 


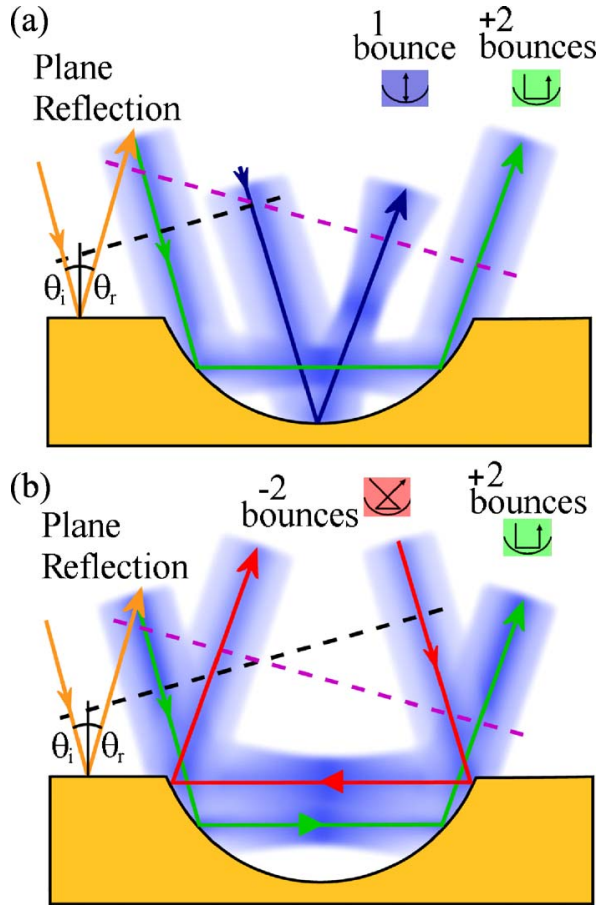

FIG. 11. (Color online) Schematic ray paths through the voids with (a) increasing bounce number and (b) different bounce directions.

(within a range of angle $\Delta \theta$ set by the experimental collection geometry) will influence the observed reflectivity, so all other ray paths are discarded. This leaves contributions from rays of integer numbers of bounces through the cavity ${ }^{14}$ as well as a reflection from the planar top surface. The cavity can support ray paths which travel in either propagation direction through the void, defined as positive when the interior bounce is parallel to the in-plane component of $k_{i}$ and negative when the bounce is in the opposite direction [Fig. 11(b)]. For each ray path the total distance between the phase front incident on the surface (black dashed line) and the phase front leaving the surface [purple (gray) dashed line] is computed. If light of a specific wavelength illuminates the cavity each ray path acquires a different phase. The interference between these ray paths leads to a modulation in the total observed reflectivity. Paths with more than two bounces have a negligible cross section, so can be ignored. Performing this calculation in three dimensions accounting for the solid angles of each bounce and the additional phase change on reflection, allows a set of theoretical absorption spectra to be produced.

An unknown quantity in this model is the Gouy shift, the phase change that occurs as a beam of light passes through a focus. ${ }^{18,19}$ When the total phase change through the focus is summed from $z=-\infty$ to $+\infty$ it equals $\pi$. For a Gaussian beam profile, this phase change occurs mainly within a distance equal to the beam waist. Since this distance is greater than the distance the light travels through the cavity, only a fractional phase change is expected. Estimating this Gouy shift is not well grounded for subwavelength propagation; hence we vary this fixed additional Gouy phase shift contribution to the interference in order to best match the experimental data.

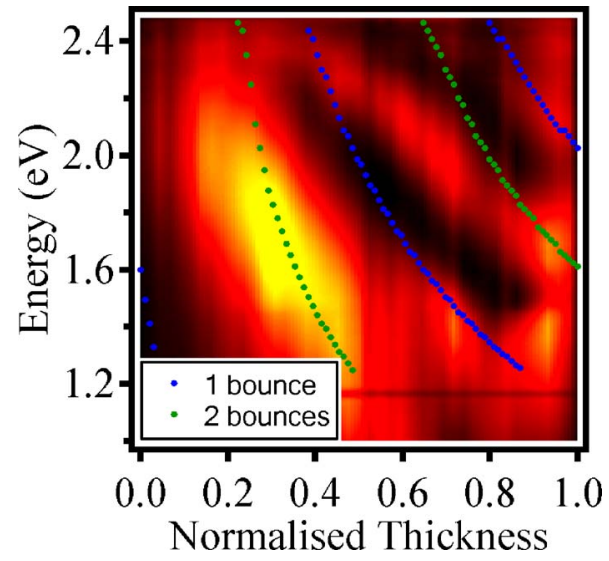

FIG. 12. (Color online) Absorption of randomly arranged voids (from Fig. 8) with predicted absorption peak energies at different thicknesses from the ray optics model.

The ray optics predictions vs void thickness shown in Fig. 12 produce a trend of decreasing mode energy with increasing thickness, as also seen experimentally in the absorption data for randomly arranged voids (shown superimposed). In this figure a Gouy shift of $0 \pi$ and $0.4 \pi$ is used for one bounce and two bounces, respectively. This can be understood since a ray path undergoing one bounce is only weakly focused while $40 \%$ of a full Gouy shift is consistent with the beam focusing that a two-bounce ray would experience on its transit through the micromirror.

However, there is only a weak agreement between ray theory and experiment. The minima from the multiple interference of rays around $\bar{t}=1$ roughly coincide with the Mie plasmon energies due to the fitting of the Gouy shift. Including both single and double bounce ray paths produces a mode separation roughly in agreement with experiment. On the other hand, for small thicknesses the interference model modes tend to infinite photon energy instead of converging to the $2 \mathrm{D}$ surface plasmon. In addition, the gradient $d E / d \bar{t}$ of the modes is greater than that in the experimental data.

A further aspect predicted by the ray optics model is that of ray clipping. This occurs either when ray paths cannot exist within a cavity (multiple bounces when the film thickness is very thin) or when the cavity rim blocks bounces around the cavity (multiple bounces when the cavity is very close to $\bar{t}=1$ ). The normalized thicknesses that allow rays of particular incident angle and number of bounces to escape are shown in Fig. 13(a). Here, primary colours correspond to ray paths of $+1,+2$, and -2 bounces, while mixtures of colors represent regions of the parameter space where more than one ray path is allowed. Only at high incident angles and normalized thicknesses close to $\bar{t}=1$ are all ray paths forbidden. Experimental absorption data plotted in the same manner are shown in Fig. 13(b) for two samples of void diameters 700 and $900 \mathrm{~nm}$. These data are averaged over all observed energies to reduce the effect of Bragg plasmons. Clearly apparent in both images is a strong band of absorption from $\theta=20^{\circ}$ to $70^{\circ}$ to centered around $\bar{t}=0.4$. While this roughly corresponds to the predicted region where -2 bounce ray paths are allowed, the agreement is not compelling. 

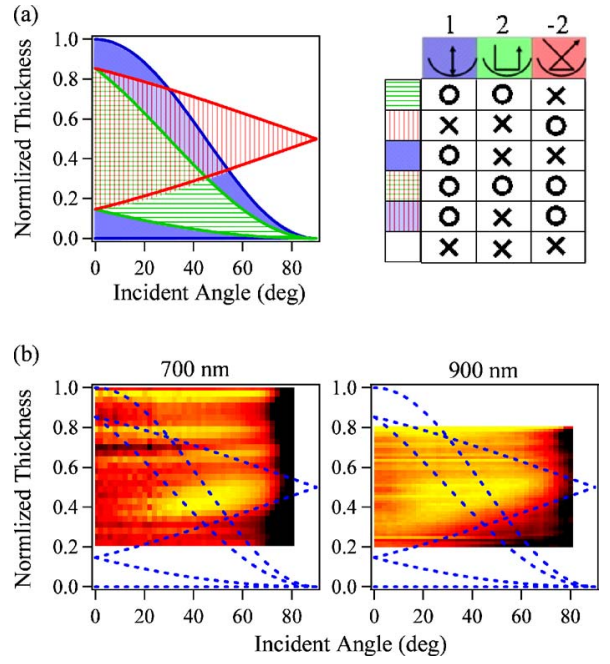

FIG. 13. (Color online) (a) Predicted regions of allowed ray paths for $1,+2$, and -2 bounces in the parameter space $(\bar{t}, \theta)$. (b) Corresponding integrated data from samples with void diameters 700 and $900 \mathrm{~nm}$, plotted as in (a).

\section{Standing wave plasmon model}

An alternative approach to modeling the plasmon modes of a truncated void is to assume the existence of surface plasmon-polariton standing waves within the dish. Plasmons are assumed to propagate in the void as on flat gold, but are reflected from the sharp upper rim. In our recent work on pyramidal pits, this model is found to account well for the localized plasmon energies. ${ }^{12,20}$ This plasmon standing wave can consist of an arbitrary number of nodes and is pinned at the void rims (Fig. 14).

A one-dimensional model is used to calculate the wave vector of the standing wave plasmon $k_{\text {swp }}$ using

$$
k_{s w p}=p \frac{2 \pi}{y}=\frac{\pi p}{D_{\text {void }} \arccos (1-2 \bar{t})}
$$

where $p$ is an integer and corresponds to the number of half wavelengths that will fit into the curved rim-to-rim distance $y$. As depicted, at normal incidence only asymmetric standing waves can be excited (even $p$ ). Subsequent use of the surface plasmon dispersion relation leads to the equation

$$
E=\hbar c k_{s w p} \sqrt{\epsilon_{d}^{-1}+\epsilon_{m}^{-1}(E)}
$$

where as before, $\epsilon_{m}$, and $\epsilon_{d}$ are the dielectric functions of the metal and dielectric. These equations provide a set of ener-

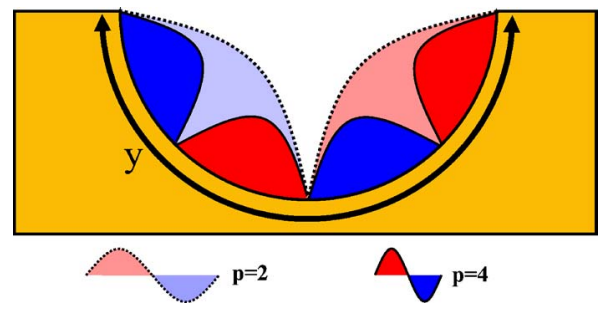

FIG. 14. (Color online) Plasmon standing wave model for the first two modes.

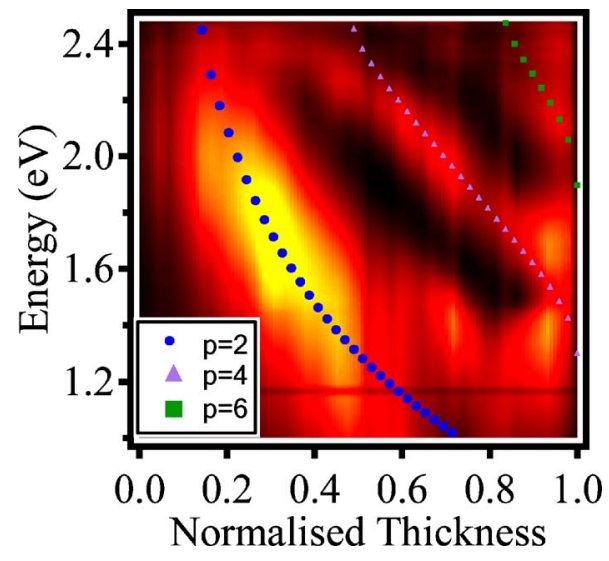

FIG. 15. (Color online) Plasmon standing wave model vs void thickness for $p=2,3,4$ superimposed on the experimental absorption of random voids from Fig. 7.

gies for the localized plasmons purely in terms of the void diameter and sample thickness and ensure that the modes are plasmonic. Solutions to the standing wave equations for a $600 \mathrm{~nm}$ cavity are shown in Fig. 15 superimposed on the experimental absorption for a sample of random voids. A surprisingly strong correlation is found between the standing wave plasmon model and the data. In this simple model there is no explicit angular momentum; however, the standing wave nodes $p=2,3$ match the Mie modes $l=1,2$. Clearly for $\bar{t}>0.8$ the theory is not consistent with the data for the reasons discussed above.

Summarizing this section, two simple models are presented which provide similar trends to the experimental data. A better account of the spectra comes from a 1D plasmon standing wave model, which also accounts for void plasmons in pyramidal pits of different size. ${ }^{12,20}$ Hence it appears that plasmon standing waves provide a good first approximation for estimating the energies of localized plasmons.

\section{PLASMON MIXING}

The energy of Bragg modes varies as the incident and azimuthal angles are varied at any point on the sample (Sec. III), while the Mie plasmon energies can be controlled by varying the thickness (Sec. IV). This provides ample control to bring the two types of plasmons into resonance with one another and observe how they interact. ${ }^{8,21}$ This coupling has been treated in detail theoretically by Teperik et al. ${ }^{15,16}$ Here corresponding experimental evidence is provided for several situations.

\section{A. Thick films}

On thick samples the Mie plasmons are well defined, and Bragg plasmons propagate on the top surface above them. At a thickness of $\bar{t}=1.3$ [Fig. 16(a)], the voids are almost completely encapsulated, turning off excitation of Mie plasmons and producing weak Bragg plasmons whose dispersion follows Eq. (1). As the holes giving access to the voids are slightly widened $(\bar{t}=1.1)$, coupling between Mie and Bragg plasmons is clearly observed [Figs. 16(b)-16(e)] in both TE 

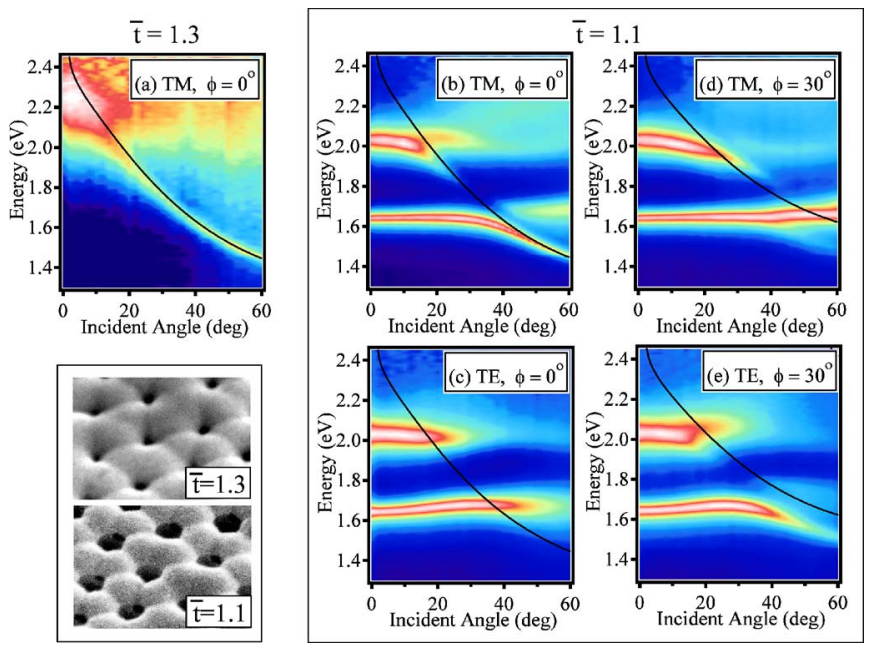

FIG. 16. (Color online) Dispersion plots for a gold sample with void diameters of $500 \mathrm{~nm}$ at thicknesses of $\bar{t}=$ (a) 1.3 and (b)-(e) 1.1 for polarization and $\phi$ as labeled. Inset: SEM images of the sample surfaces.

and TM polarizations. At this thickness, two strongly localized Mie plasmons are present corresponding to the $l=1$ mode (at $1.64 \mathrm{eV}$ ) and $l=2$ mode (at $2.03 \mathrm{eV}$ ). At most energies Bragg plasmons are only very weakly excited because the surface corrugations (shown in the SEMs) are only fractions of an optical wavelength. However at energies for which Bragg and Mie modes are in resonance, the Bragg plasmons become strongly excited due to the large optical cross section of the Mie modes. ${ }^{14,22}$

This coupling is sufficiently strong to produce anticrossings under some conditions, indicating that new mixed mode plasmons are formed. These are based on linear combinations of the localized $\left(\psi_{L P}^{(l)}\right)$ and $\operatorname{SPP}\left(\psi_{S P P}\right)$ :

$$
\psi_{ \pm}=\frac{1}{\sqrt{2}}\left\{c_{1} \psi_{L P}^{(l)} \pm c_{2} \psi_{S P P}\right\}
$$

Such states correspond to the bonding and antibonding states between plasmon atoms confined in the voids, and plasmon bus states on the upper surface. ${ }^{8}$ The smaller absorption observed for the antibonding (higher-energy) mixed plasmons must be due to their decreased overlap with incident plane waves, possibly as they possess more field nodes. Stronger coupling (roughly three-fold) is observed at $l=1$ than $l=2$ due to the greater overlap of the electric fields betwen the top surface and the more delocalized $l=1$ Mie plasmon (Fig. 7). The coupling also depends on incident light polarization and sample orientation, and is maximized for TM $\left(\phi=0^{\circ}\right)$ and TE $\left(\phi=30^{\circ}\right)$. This can be understood by considering the field directions of Bragg and Mie plasmons. For Mie plasmons, the plasmon polarization is oriented along the incident electric field. For a Bragg plasmon traveling along in-plane wave vector $\mathbf{q}_{m n}$, the plasmon field has components both along $\mathbf{q}$ and perpendicular to the surface. Examining Fig. 3 shows that the relevant lowest-energy Bragg plasmons (thick lines) have a $\mathbf{q}$ parallel to the incident field only for exactly the observed resonant orientations
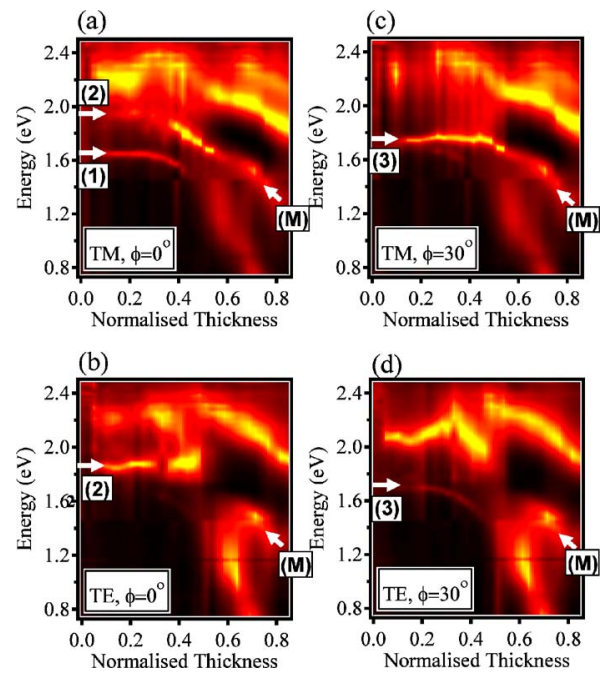

FIG. 17. (Color online) Absorption spectra at fixed incident angle of $\theta=20^{\circ}$ showing photon energy against normalized thickness for a sample with a void diameter of $600 \mathrm{~nm}$ for (a) TM $\phi$ $=0^{\circ}$, (b) TE $\phi=0^{\circ}$, (c) TM $\phi=30^{\circ}$, (d) TE $\phi=30^{\circ}$. The white arrows indicate Bragg plasmons excited with wave vectors of (1) $q_{11}^{-}$, (2) $q_{10}^{\overline{1}}, q_{0 \overline{1}}$, (3) $q_{1 \overline{1}}^{\overline{1}}, q_{0 \overline{1}}$, and (M) $l=1$ Mie plasmon.

above, wheras the opposite input polarizations have a perpendicular alignment. Hence, as expected, mixed plasmons are only observed when Mie and Bragg fields have some common field component.

\section{B. Thin films}

For a particular void thickness it is possible to alter the Bragg plasmon energies by varying the azimuthal orientation of the incident light. This degree of freedom allows the interactions between different Bragg plasmons with a single Mie plasmon to be resolved. This is evident in Fig. 17 which shows the dependence of plasmon energies on increasing sample thickness at fixed incident angle of $\theta=20^{\circ}$ at sample orientations of $\phi=0^{\circ}$ and $30^{\circ}$ for TM and TE polarizations.

We focus on the interactions between the $l=1$ Mie mode, $\psi_{L P}^{1}$ (white arrows labeled by $\mathrm{M}$ ) and the three lowest-energy Bragg modes (white arrows labeled by numbers) excited with wave vectors of (1) $q_{11}^{\overline{1}}$, (2) $q_{10}^{-}, q_{01}$, and (3) $q_{11}^{-\overline{1}}, q_{01}$. For the thin region of the sample $(\bar{t}<0.3)$ only pure Bragg modes are observed below $2 \mathrm{eV}$. As the thickness increases $(0.25<\bar{t}<0.45)$ some of the Bragg modes split and drop in energy. At greater thicknesses $(\bar{t}>0.4)$ the Bragg modes encounter the redshifting Mie mode, resulting in either mixing or their disappearance.

To understand these phenomena we plot the standing waves making up the Bragg plasmons (Fig. 18) which are excited by different polarizations (using the coupling arguments developed above). ${ }^{21}$ For mode (1), the Bragg plasmon is completely delocalized over the upper surface. On the other hand for modes (2) and (3) the Bragg plasmons form Bloch waves with standing wave and propagating components. Taking the time average of these over one optical cycle produces the standing wave intensity distributions 


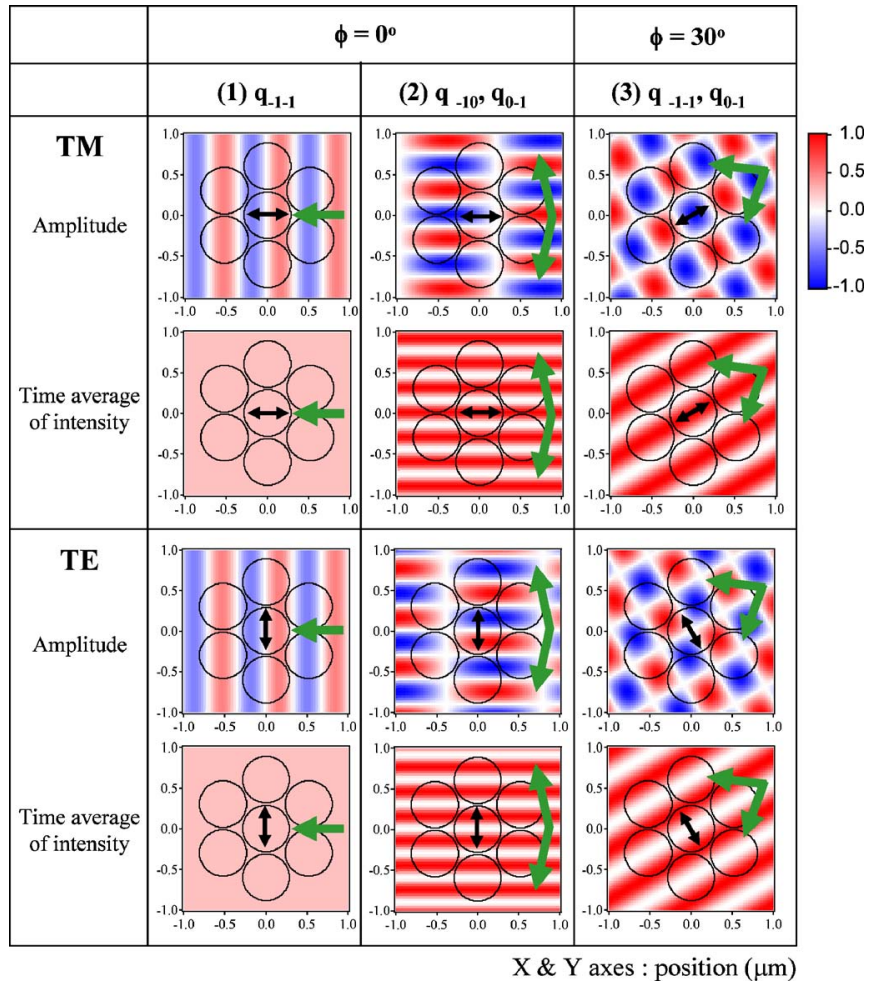

FIG. 18. (Color online) Predicted amplitudes and intensities of optical fields for the lowest-energy Bragg plasmons showing the plane wave superpositions. The $x$ and $y$ axes are in micrometers, intensities normalized to 1 . Black arrows show dominant field polarization of Mie modes, green (gray) arrows show dominant inplane field polarization of Bragg wave components.

shown, which shift by half a period as the polarization is flipped.

We discuss each situation in turn to give an intuitive picture of the coupling. For TM $\left(\phi=0^{\circ}\right)$, Bragg mode (1) overlaps with $\psi_{L P}^{1}$ producing strongly coupled bonding and anti bonding mixed plasmons. The lower-energy (bonding) mixed plasmon $\psi_{+}$is delocalized over the entire surface (hence dropping in energy), while the upper (antibonding) mode $\psi_{-}$ is localized both within the voids and between them. While the former becomes the $l=1$ Mie plasmon, the latter disappears as the top surface breaks up at $\bar{t}=0.5$. Similarly, Bragg mode (2) splits into a component overlapping strongly with $\psi_{L P}^{1}$ and a component on the upper surface (which disappears).

For TE $\left(\phi=0^{\circ}\right)$ the Bragg standing wave of mode (2) is shifted to the upper sides of each dish, with the wrong symmetry now to couple to $\psi_{L P}^{1}$ (see Fig. 7). Hence the mode vanishes at $\bar{t}=0.5$. Bragg mode (1) has perpendicular field polarization to the incident field, and hence is not visible.

For TM $\left(\phi=30^{\circ}\right)$ the Bragg standing wave of mode (3) is completely centred in the voids, matching completely the mode symmetry and position of $\psi_{L P}^{1}$, into which it transforms. No strong coupling is seen because $\psi_{-}$is very small since the basis modes of which it is composed are almost identical.

For TE $\left(\phi=30^{\circ}\right)$ this Bragg standing wave is shifted onto the narrow strips of metal surface in-between the voids. As these reduce in size, the mode drops in energy and vanishes at $\bar{t}=0.4$

Hence a reasonable account of the mode coupling and its dependence on polarization and orientation is provided by considering the distributions of standing waves (in the empty lattice approximation) and Mie plasmons. More accurate comparisons await theories capable of handling both Bragg and Mie plasmons in this plasmonic architecture.

\section{Full thickness dependence}

In the previous sections particular features are extracted from the four-dimensional data set $R(\theta, \phi, \bar{t}, \lambda)$ to highlight how it can be used to identify the type, symmetry, and coupling of the different plasmons. However a wealth of further complexity exists in our measurements, and hence a complete data set is presented for one particular gold sample with $D=600 \mathrm{~nm}$ (representative of the many samples analyzed). Copolarized absorption are shown in Fig. 19 for TE and TM polarizations at $\phi=0^{\circ}$ and $30^{\circ}$ (cross-polarized data show an increased complexity of mode mixing but with $<1 \%$ intensity). The slight discontinuity in the images near $1.5 \mathrm{eV}$ is due to stitching between data from visible and infrared spectrometers, while the sharp line at $1.2 \mathrm{eV}$ is a remnant of the pump laser used to produce the supercontinuum. The normalized thickness for each position has been actively determined through analysis of SEM images.

Different thickness regimes can be identified.

$\bar{t}=0-0.4$ : Bragg plasmons are seen, becoming increasingly strong as the corrugation deepens. The Bragg mode Bloch waves are increasingly split by the stronger scattering potential. The lowest Bragg mode at $k=0$ is invisible due to the symmetry of the surface. ${ }^{23}$ The Bragg modes disappear as the top surface breaks up into islands.

$\bar{t}=0.4-0.9$ : Mie plasmons appear at high energies around $\bar{t}=0.35$, continuously redshifting at increasing thickness. Mie modes are almost independent of azimuthal angle (as they are localized). They are also visible for TE excitation at specific angles of incidence. Eventually for this $D$, two Mie plasmons are observed dropping down from high energy. The strong coupling as they mix with the Bragg modes is clearly visible, creating complex dispersion relations.

$\bar{t}=0.9-1.1$ : As the sample thickness reaches void encapsulation the Mie plasmon modes become better confined within the cavities. This leads to clear localized plasmons at the expected energies. Bragg plasmons reappear and coexist on the top surface of the film, coupling with these Mie plasmons.

In addition, around $\bar{t}=0.54-0.76$, additional absorption bands are observed. These start out as strong spectrally broad but angularly localized resonances around $\theta=65^{\circ}$ visible in TM polarization [labelled by (a)] and a spectrally and angularly localized resonance around $\theta=65^{\circ}, E=1.65 \mathrm{eV}$ visible in TE polarization [labeled by (b)]. Subsequently an angularly broad, spectrally narrow low-energy mode [labeled by (c)] visible in both polarizations drops below the $l=1 \mathrm{Mie}$ plasmon and redshifts by $\bar{t}=0.64$ below our spectral range. The appearance of this low-energy mode coincides with the formation of the small holes between the neighboring spheri- 

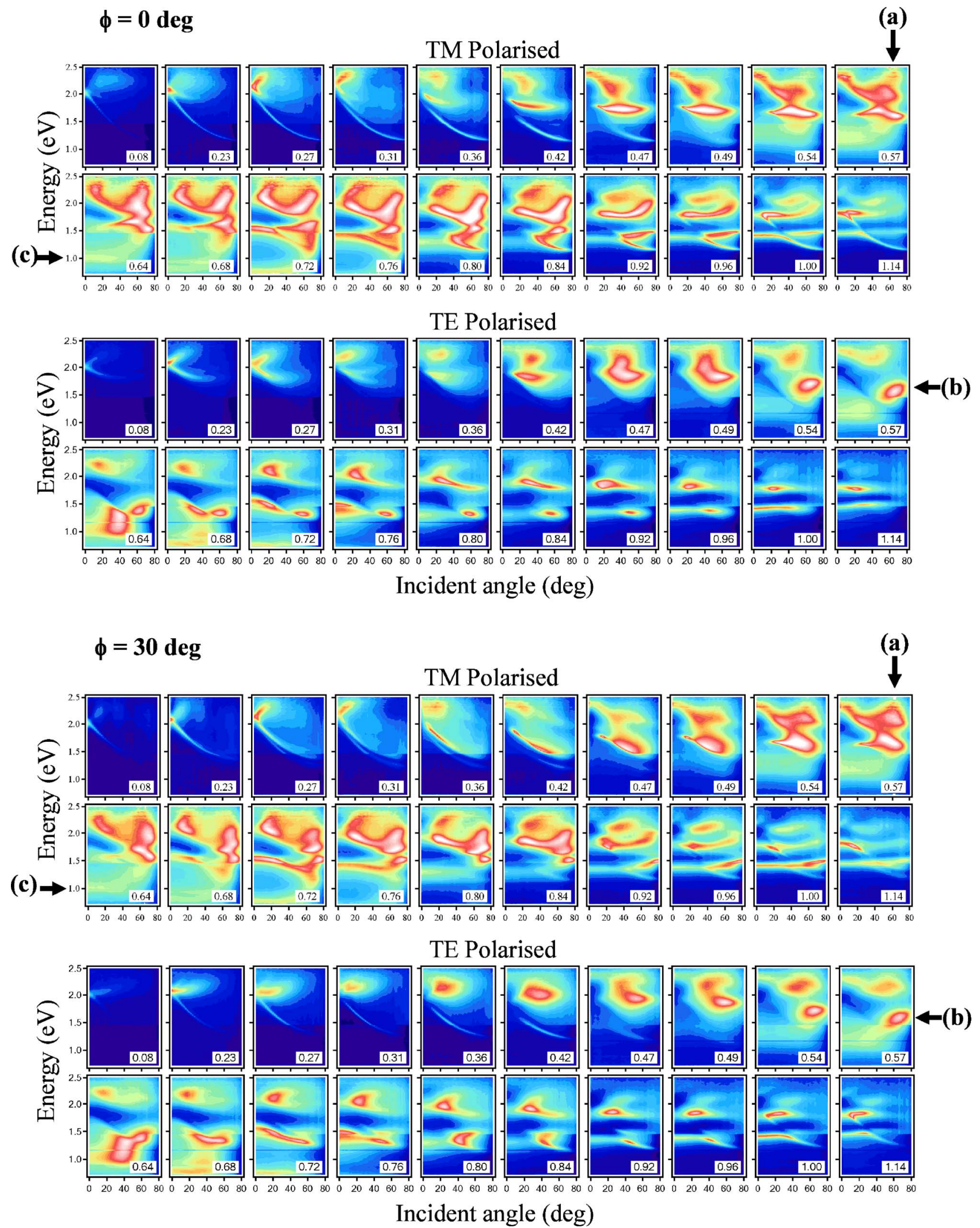

FIG. 19. (Color online) Complete dispersion maps for increasing void thicknesses $\bar{t}=0.08-1.14$ on a gold sample with void diameters of $600 \mathrm{~nm}$. Data sets with TM and TE polarizations and $\phi=0^{\circ}, 30^{\circ}$ are presented for photon energies from 0.7 to $2.5 \mathrm{eV}$ and incident angles from $0^{\circ}$ to $80^{\circ}$. Color (gray) scale (logarithmic to enhance weaker features) is from blue (black) (maximum reflectivity) to white (minimum reflectivity). 


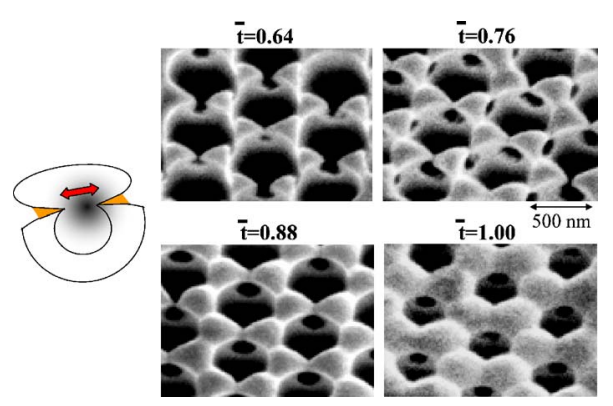

FIG. 20. (Color online) FESEM images of the sample at normalized thicknesses of $\bar{t}=0.64,0.76,0.88$, and 1.00 . Two levels of magnification are shown to better emphasize the geometry of the surfaces.

cal voids and is visible for all our close-packed samples. On samples in which the voids are spaced further apart, this mode is indeed found to be eliminated.

Higher-resolution field-emission scanning electron micrographs shown in Fig. 20 illustrate the complex morphology of the gold surface in this thickness range around half-sphere height. Electrochemical growth within the space between the latex spheres (which are $50 \mathrm{~nm}$ apart) is locally switched off by screening from the higher deielectric polymer, leaving a hole to form. This breaks up the top surface into triangular islands which grow in pairs into tetrahedral pillars. These reconnect around $\bar{t}=0.65$ forming bowtie-shaped features above a circular window between the voids. We believe that these features support additional localized plasmons, ${ }^{24}$ and further experiments and analysis are currently under way.

\section{CONCLUSIONS}

In this paper, a comprehensive account of the plasmons supported by metal films containing embedded truncated metal voids is provided. Detailed experiments mapping the dispersion relation are shown to be crucial in identifying the presence of both localized and delocalized plasmons, and their mixing when resonant. We successfully model Bragg plasmons in a weak-scattering limit for thin films. Localized Mie plasmons are seen at larger thicknesses and their spectral tuning accounted for using a number of intuitive models. A combination of analytic solutions in the complete voids, and standing wave models of surface plasmon-polaritons, allow good comprehension of the data. Strong coupling between the plasmons is observed, and shown to depend on the orientation of sample and incident fields. The richness of the plasmon modes for different geometries are evident in their complete absorption dispersions. Given the applications recently demonstrated for surface-enhanced Raman scattering ${ }^{4,25-27}$ on such nanovoid surfaces, it remains critical to build more detailed understanding of the nature of their plasmons. However, the work presented here provides the essential phenomenology of void plasmons.

\section{ACKNOWLEDGMENTS}

We gratefully acknowledge many fruitful discussions with T. Teperik. We would also like to thank J. F. Garcia de Abajo and V. Popov for useful discussions. This work was supported by EPSRC Grant No. EP/C511786/1. Y.S. was supported by JSPS.
*Electronic address: j.j.baumberg@ soton.ac.uk

${ }^{1}$ W. Barnes, A. Dereux, and T. Ebbesen, Nature (London) 424, 824 (2003).

${ }^{2}$ A. Krasavin, A. Zayats, and N. Zheludev, J. Opt. A, Pure Appl. Opt. 7, S85 (2005).

${ }^{3}$ A. Zayats and I. Smolyaninov, J. Opt. A, Pure Appl. Opt. 5, 16 (2003)

${ }^{4}$ J. Baumberg, T. Kelf, Y. Sugawara, S. Cintra, M. Abdelsalam, P. Bartlett, and A. Russell, Nano Lett. 5, 2262 (2005).

${ }^{5}$ J.-C. Weeber, J. R. Krenn, A. Dereux, B. Lamprecht, Y. Lacroute, and J. P. Goudonnet, Phys. Rev. B 64, 045411 (2001).

${ }^{6}$ T. Ebbesen, H. Lezec, H. Ghaemi, T. Thio, and P. Wolff, Nature (London) 391, 667 (1998).

${ }^{7}$ H. Lezec and T. Thio, Opt. Express 12, 3629 (2004).

${ }^{8}$ T. A. Kelf, Y. Sugawara, J. J. Baumberg, M. Abdelsalam, and P. N. Bartlett, Phys. Rev. Lett. 95, 116802 (2005).

${ }^{9}$ P. Bartlett, J. Baumberg, P. Birkin, M. Ghanem, and M. Netti, Chem. Mater. 14, 2199 (2002).

${ }^{10}$ P. Bartlett, J. Baumberg, S. Coyle, and M. Abdelsalam, Faraday Discuss. 125, 117 (2003).

${ }^{11}$ P. Johnson and R. Christy, Phys. Rev. B 6, 4370 (1972).

${ }^{12}$ N. M. B. Perney, J. Baumberg, M. E. Zoorob, M. D. B. Charlton, S. Mahnkopf, and C. M. Netti, Opt. Express 14, 847 (2005).

${ }^{13}$ A. Boardman, Electromagnetic Surface Modes (John Wiley and Sons, New York, 1982).

${ }^{14}$ S. Coyle, M. C. Netti, J. J. Baumberg, M. A. Ghanem, P. R. Birkin, P. N. Bartlett, and D. M. Whittaker, Phys. Rev. Lett. 87,
176801 (2001)

${ }^{15}$ T. V. Teperik, V. V. Popov, and F. J. Garcia de Abajo, Phys. Rev. B 71, 085408 (2005); Phys. Status Solidi A 202, 362 (2005); Phys. Solid State 47, 178 (2005).

${ }^{16}$ T. V. Teperik, V. V. Popov, F. G. Garcia de Abajo, M. E. Abdelsalam, P. N. Bartlett, T. A. Kelf, Y. Sugawara, and J. J. Baumberg, Opt. Express 14, 1965 (2006).

${ }^{17}$ S. Coyle, G. Prakash, J. J. Baumberg, M. E Abdelsalam, and P. N. Bartlett, Appl. Phys. Lett. 83, 767 (2003).

${ }^{18}$ T. Ackemann, W. Grosse-Nobis, and G. Lippi, Opt. Commun. 189, 5 (2001).

${ }^{19}$ P. Hariharan and P. Robinson, J. Mod. Opt. 43, 219 (1996).

${ }^{20}$ N. M. B. Perney, J. J. Baumberg, M. E. Zoorob, M. D. B. Charlton, and C. M. Netti (unpublished).

${ }^{21} \mathrm{~T}$. V. Teperik (private communication).

${ }^{22}$ A. Lucas, Phys. Rev. B 7, 3527 (1973).

${ }^{23}$ W. L. Barnes, T. W. Preist, S. C. Kitson, and J. R. Sambles, Phys. Rev. B 54, 6227 (1996).

${ }^{24}$ P. J. Schuck, D. P. Fromm, A. Sundaramurthy, G. S. Kino, and W. E. Moerner, Phys. Rev. Lett. 94, 017402 (2005).

${ }^{25}$ S. H. Cintra, M. E. Abdelsalam, P. N. Bartlett, J. J. Baumberg, T. A. Kelf, Y. Sugawara, and A. E. Russell, Faraday Discuss. 132, 191 (2005).

${ }^{26} \mathrm{http}: / / \mathrm{www} . \mathrm{mesophotonics.} \mathrm{com}$

${ }^{27}$ J. J. Baumberg et al., University of Southampton, U. K. Patent No. 0420158.8 (2006). 